\title{
Cuantificación de Sapogeninas del Jugo Fresco y Fermentado de Fique (Furcraea gigantea) mediante Cromatografía Liquida de Alta Resolución (HPLC-PDA)
}

\author{
Olga L. Benavides, Oscar Arango, Andrés M. Hurtado, Myriam C. Rojas \\ Universidad de Nariño, Grupo de Investigación Tecnologías Emergentes en Agroindustria (TEA), \\ Facultad de Ingeniería Agroindustrial, Calle 18 Carrera 50, Ciudad Universitaria Torobajo, Pasto \\ (Nariño), Colombia (e-mail: olgalucia@udenar.edu.co).
}

Recibido Ago. 25, 2011; Aceptado Oct. 21, 2011; Versión final recibida Dic. 21, 2011

\begin{abstract}
Resumen
Se presenta un estudio sobre la cuantificación de sapogeninas del jugo fresco y fermentado de fique (Furcraea gigantea) mediante cromatografía liquida de alta resolución con detector de fotodiodo (HPLC-PDA). El jugo de fique, una planta cultivada ampliamente en Colombia, se fermentó durante 2, 4 y 6 días a $33^{\circ} \mathrm{C}$. Mediante hidrólisis ácida se obtuvo el extracto de sapogeninas, cuyos componentes identificados por cromatografía gaseosa y espectroscopia másica (GC-MS) fueron hecogenina y tigogenina. Usando el método HPLC-PDA se cuantificó la hecogenina y tigogenina presentes. El jugo de fique con cuatro días de fermentación presentó el mayor contenido de sapogeninas. Por lo tanto, se recomienda este proceso para fines de extracción de estos compuestos.
\end{abstract}

Palabras clave: sapogeninas, hecogenina, tigogenina, HPLC-PDA, GC-MS, Furcraea gigantea

\section{Sapogenins Quantification of Fresh and Fermented Juice of Fique (Furcraea gigantean) by High- Performance Liquid Chromatography (HPLC-PDA)}

\begin{abstract}
A study on the quantification of sapogenins in fresh and fermented juice of fique (Furcraea gigantean) by high-performance liquid chromatography with photodiode array detector (HPLCPDA). The juice of the fique, a plant that is widely grown in Colombia, was fermented for 2, 4 and 6 days at $33^{\circ} \mathrm{C}$. Using acid hydrolysis, sapogenin extracts were obtained. The substances in the extracts, identified by gas chromatography-mass spectrometry (GC-MS) were hecogenin and tigogenin. Hecogenin and tigogenin in the juice were determined using HPLC-PDA. Fique juice with four days of fermentation showed the highest content of sapogenins. Therefore, this is the recommend process for the extraction of these compounds.
\end{abstract}

Keywords: sapogenins hecogenin, tigogenin, HPLC-PDA, GC-MS, Furcraea gigantea 


\section{INTRODUCCIÓN}

El fique (Furcraea spp.) es una planta perteneciente a la familia Agavaceae de la cual se obtiene una fibra con la que se elaboran empaques naturales, cordeles, sogas, geotextiles y artesanías, entre otros. En Colombia el área cultivada es superior a las 23.000 hectáreas, con una producción aproximada de 18.750 toneladas de fibra de fique al año, la cual representa únicamente el $4 \%$ del peso de la hoja; el restante $96 \%$ (aproximadamente $450.000 \mathrm{t} \mathrm{a}^{-1}$ ) son desechos industriales $(70 \%$ jugo y $26 \%$ bagazo), que son arrojados sin ningún tratamiento a suelos y fuentes hídricas en zonas rurales (MADR \& MAVDT, 2006). Estos residuos, en especial el jugo de fique, constituyen un desecho altamente contaminante y tóxico para los peces y los organismos acuáticos, debido a su contenido de azúcares y otros compuestos como saponinas y alcaloides (Martínez y Caicedo, 2002). Los productores de fique en Colombia constituyen un sector que vive bajo la línea de pobreza por lo que la obtención de productos con alto valor agregado a partir de los residuos del beneficio de la planta podría significar una mejora sustantiva en sus condiciones económicas.

Mientras en Colombia las pencas de fique son empleadas para la producción de fibras naturales, en países como México se emplean las cabezas de las Agavaceas para la producción de mezcal (Durán y Pulido, 2007). El jugo hidrolizado de las pencas de Agave tequilana presenta un importante contenido de azúcares fermentables, por lo cual ha sido estudiado en la producción de bioetanol como alternativa para el aprovechamiento integral del cultivo (Montañez et al., 2011).

Las saponinas son moléculas de estructuras diversas denominadas químicamente como triterpenos y glicósidos esteroidales que consisten en agliconas no polares unidas con una o más fracciones de monosacáridos (Oleszek y Bilaly, 2006); esta combinación de elementos polares y no polares explican su comportamiento como jabón en soluciones acuosas. Debido a sus propiedades como agente espumante las saponinas son utilizadas en la industria cosmética y farmacéutica (San Martin and Briones, 1999). La mayoría de las sapogeninas exhiben actividades farmacológicas, lo que las ha convertido en objeto de estudio para el desarrollo de nuevos medicamentos (Augustin et al., 2011), entre tales actividades se encuentran efectos antiinflamatorios (Sun et al., 2010; Tapondjou et al., 2008) anticancerogénicos (Musende et al., 2009; Man et al., 2010), antibacteriales, antifúngicos y antivirales (De Leo et al., 2006; Saleem et al., 2010; Coleman et al., 2010; Rattanathongkom et al., 2009). Fundamentalmente las sapogeninas se han constituido desde hace bastante tiempo como precursores únicos de muchos medicamentos esteroides tales como hormonas sexuales (Hostettmann and Marston, 1995). Las sapogeninas son consideradas como una alternativa de excelentes posibilidades comerciales y de industrialización debido a su alto precio en el mercado el cual puede variar de acuerdo a su nivel de pureza desde 6 hasta 142 dólares por gramo de producto (MADR \& MAVDT, 2006).

La familia Agavaceae es conocida por ser fuente importante de saponinas esteroidales. Blunden et al., (1980) estudiaron extractos de hojas de 7 especies de agave y 1 especie de Furcraea, en todas ellas la hecogenina y tigogenina fueron las principales sapogeninas aisladas. Recientemente, Yokosuka y Mimaki (2009) aislaron 15 saponinas esteroidales a partir de Agave utahensis.

El análisis de sapogeninas mediante HPLC con detectores tipo UV es un proceso complejo, ya que las sapogeninas son moléculas con baja absorbancia lo que dificulta su detección; por lo tanto se hace necesario su derivatización, es así como Higgins (1976), derivatizó la hecogenina de una agavácea empleando cloruro de benzoílo y obtuvo un cromóforo posible de analizar. En el caso de no realizar la derivatización, es viable utilizar otro tipo de detectores. De esta forma Sitton et al., (1982) analizaron sapogeninas de agave utilizando un detector de Índice de Refracción (IR) o bien, Oleszek y Bilaly (2006) caracterizaron sapogeninas con detector ELSD. Eskander et al., (2010) emplearon cromatografía líquida en fase reversa para la separación de las fracciones de saponinas de las hojas de Agave macroacantha y aislaron un nuevo compuesto además de 3 conocidos, en los cuales, la aglicona resultó ser hecogenina.

Hasta el momento no se han encontrado publicaciones sobre el análisis de sapogeninas procedentes del jugo de fique (Furcraea gigantea) fermentado a diferente tiempo, razón por la cual 
el objetivo de este trabajo consistió en determinar la presencia de las sapogeninas hecogenina y tigogenina en este producto utilizando HPLC con detector de arreglo de diodos (PDA).

\section{MATERIALES Y MÉTODOS}

\section{Material vegetal}

Se recolectaron hojas de Furcraea gigantea de aproximadamente 15 años de edad, procedentes de la zona rural del municipio de El Tambo, Departamento de Nariño (suroccidente de Colombia). Las hojas fueron cortadas al amanecer, se eliminaron sus espinas y se transportaron a la Planta Piloto de la Facultad de Ingeniería Agroindustrial de la Universidad de Nariño. El tiempo transcurrido entre el corte y el inicio del proceso de extracción del jugo fue de 3 horas. La identificación taxonómica de la planta fue realizada en el Herbario de la Universidad de Nariño.

\section{Reactivos y solventes}

Para el proceso de hidrólisis se utilizó $\mathrm{HCl}$ (Merck) al 37\%, para la extracción líquido-líquido se usó $\mathrm{CHCl}_{3}$ absoluto (Merck, Alemania), la cristalización se hizo con $\mathrm{MeOH}$ absoluto grado HPLC (Fisher, USA), para las filtraciones se usó papel filtro cualitativo y cuantitativo (Boeco, Alemania). En la fase móvil cromatográfica se empleó $\mathrm{H}_{2} \mathrm{O}$ grado HPLC (Fisher, USA) y MeCN grado HPLC (Fisher, USA).

Los estándares de sapogeninas utilizados fueron ácido oleanólico al $98 \%$ y acetato de hecogenina al 90\% marca Sigma (Sigma Aldrich, USA).

\section{Obtención y acondicionamiento de las muestras de jugo de fique}

Se obtuvo el jugo fresco exprimiendo las hojas de $F$. gigantea en un molino de rodillos de 2,5 HP de potencia (Zutta Hnos, Pasto, Colombia). El líquido obtenido se filtró mediante un tamiz con malla de $2 \mathrm{~mm}^{2}$ recolectando una primera muestra de $2 \mathrm{~L}$, la cual se sometió a liofilización en un equipo marca Labconco modelo FreeZone 2.5 (Labconco Corporation, Kansas City, Missouri, USA), a una presión de $0,6 \mathrm{~mm} \mathrm{Hg}$ con temperatura escalonada de -35 a $20^{\circ} \mathrm{C}$ durante $48 \mathrm{~h}$. Se recogió una segunda muestra de jugo del mismo volumen en un recipiente de acero inoxidable, la cual se sometió a tratamiento térmico de $65^{\circ} \mathrm{C}$ por $30 \mathrm{~min}$ con ayuda de una placa de calefacción, con el fin de evitar el inicio del proceso de fermentación. Posteriormente esta muestra se deshidrató hasta peso constante en un secador de bandejas (FIQ Ltda, Bogotá, Colombia) con un flujo de aire de $300 \mathrm{~m}^{3} \mathrm{~h}^{-1}$ a temperatura de $40^{\circ} \mathrm{C}$. Otras muestras de jugo fresco se sometieron a fermentación aerobia por acción de la flora natural presente en el jugo a temperatura $33^{\circ} \mathrm{C}$, durante dos, cuatro y seis días. En un estudio paralelo a esta investigación realizado en la Universidad de Nariño y cuyos resultados aún no se han publicado, se identificó la microbiota presente en el jugo de fique, encontrándose 25 aislados de bacterias y 22 de levaduras, de los cuales mediante perfiles bioquímicos se lograron identificar 14 cepas de bacterias del género Bacillus y una del género Brevibacillus, así como también 13 cepas de levaduras de los géneros Rhodotolura y Candida.

Al cabo de cada tiempo de fermentación se recolectaron 2 litros de muestra que se pasteurizó y secó en las mismas condiciones que la muestra fresca. Posteriormente, las muestras secas se molieron en mortero y se almacenaron a temperatura ambiente.

\section{Extracción de saponinas}

A partir de las muestras liofilizadas de jugo fresco y las muestras secas del jugo fermentado a diferentes tiempos, se extrajeron las saponinas con base en el método propuesto por Gnoatto (2005), empleando agua grado HPLC en relación de 1,5:20 m/v muestra: solvente. El extracto acuoso de saponinas fue filtrado en papel filtro cualitativo. 


\section{Obtención de sapogeninas}

El filtrado del extracto acuoso de saponinas se agitó en contacto con $\mathrm{HCl}$ concentrado $(1,5 \mathrm{~mL}$ de $\mathrm{HCl}$ por cada $10 \mathrm{~mL}$ de filtrado). La mezcla se sometió a reflujo durante 4 horas. Se extrajeron las sapogeninas con cloroformo en relación 1:2 respecto al hidrolizado. Se evaporó el extracto clorofórmico y el residuo obtenido correspondió al crudo de sapogeninas. Se determinó la eficiencia de la hidrólisis, con base en la masa del extracto acuoso y la masa de sapogeninas crudas. La purificación de sapogeninas se realizó por cristalización según la metodología seguida por Sharapin (2000), con algunas modificaciones; se disolvió el residuo de crudo de sapogeninas en metanol caliente empleando ultrasonido durante 15 minutos, se adicionó carbón activado y se filtró con papel filtro cuantitativo, se eliminó el metanol del filtrado y el residuo obtenido correspondió a la muestra de sapogeninas cristalizadas.

\section{Identificación de sapogeninas por GC-MS}

Se utilizó un cromatografo de gases Agilent CG 7890A FID/MSD 5975C equipado con sistema de inyección automática de líquidos, puerto de inyección capilar split splitless, sistema de detección MSD (Mass Selective Detector) de alta sensibilidad y especificidad operando en modo SCAN (40 - 700 uma). El instrumento se sintonizó en modo automático (atune), al inicio y durante el desarrollo de los análisis. Se utilizó una columna capilar DB5-MS de $30 \mathrm{~m} \times 250 \mu \mathrm{m} \times 0,25 \mu \mathrm{m}$, las temperaturas del inyector y del detector fueron 280 y $300{ }^{\circ} \mathrm{C}$, respectivamente. Se empleó una rampa de temperatura de $1 \mathrm{~min}$ a $160^{\circ} \mathrm{C}, 15 \mathrm{~min}$ a $290^{\circ} \mathrm{C}$ con un incremento de $9{ }^{\circ} \mathrm{C}$ por min. Como fase móvil se utilizó helio a $1 \mathrm{~mL} \mathrm{~min}^{-1}$.

Se tomaron 1,2 mg de sapogeninas cristalizadas y se disolvieron en $1000 \mu \mathrm{L}$ de cloroformo grado HPLC, se adicionaron $50 \mu \mathrm{L}$ en un inserto que se colocó en un vial ámbar de encapsulamiento. Se realizó una secuencia de análisis con inyección automática por duplicado. Los eventos de integración de la señal de cada analito se optimizaron y se mantuvieron para evitar la pérdida de exactitud y precisión.

\section{Cuantificación de sapogeninas por HPLC-PDA}

Una vez identificadas las sapogeninas, se procedió a la acetilación de hecogenina según el método de Bjarte (1976), mientras que para el análisis de tigogenina no se realizó acetilación. Ambas muestras se disolvieron en acetonitrilo grado HPLC mediante sonicación en un equipo de ultrasonido (Fisher) durante 15 minutos. La solución se filtró con acrodisk de 0,45 $\mu \mathrm{m}$ y se inyectó en el equipo HPLC (Cromatógrafo Breeze 2 Waters con bomba binaria 1525 y Detector PDA 2998 operado con el software Empower 2,0 (Waters, USA)).

Para el análisis por HPLC-PDA, se utilizó una columna Waters spherisorb C8 (100 mm, 4,6 mm

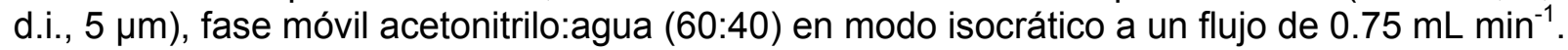

Se realizaron inyecciones de la fase móvil, del diluente del estándar y de las muestra para verificar que no hubiera interferencia por parte de éstos en el tiempo de retención del estándar, luego se inyectó cada estándar y se hizo un barrido de 200 a $400 \mathrm{~nm}$ para identificar su máxima absorbancia. Se hicieron cinco inyecciones del estándar para determinar la repetibilidad del tiempo de retención y el área de pico, este último fue el parámetro de cuantificación utilizado en esta investigación.

\section{Curva de calibración}

La cuantificación de sapogeninas del jugo de fique se realizó mediante el método de estándar externo utilizando como estándares el ácido oleanólico al $98 \%$ y acetato de hecogenina al $90 \%$ de pureza (Sigma Aldrich).

Para la curva de calibración se preparó una solución madre de 1000 ppm de cada estándar, a partir de la cual se obtuvieron diluciones de ácido oleanólico a 20, 60, 80, 100 y 120 ppm y de acetato de hecogenina a 10, 20, 40,60 y 100 ppm. De éstas, se hicieron dos inyecciones que se 
leyeron a $203 \mathrm{~nm}$ y $235 \mathrm{~nm}$, respectivamente. Los parámetros para verificar la confiabilidad del método fueron: $R^{2}, \% R S D$, resolución, factor de selectividad, así como los límites de detección y cuantificación.

La resolución y factor de selectividad, se calcularon mediante las ecuaciones 1 y 2 de Skoog, Holler \& Nieman, (2000):

$$
\begin{aligned}
& \square=\frac{\left(t_{R}\right)_{B}-t_{M}}{\left(t_{R}\right)_{A}-t_{M}} \\
& R_{s}=\frac{\left.\square 2\left[\left(t_{R}\right)\right]_{B}-\left(t_{R}\right)_{A}\right]}{W_{A}+W_{B}}
\end{aligned}
$$

\section{RESULTADOS Y DISCUSIÓN}

La eficiencia de la hidrólisis ácida fue del $87,22 \%$. De $4,5 \mathrm{~g}$ de jugo de fique liofilizado se obtuvieron 0,195 g de crudo de sapogeninas, a partir de las cuales se logró obtener 4,1 mg de sapogeninas purificadas, para un rendimiento de $0,091 \% \mathrm{p} / \mathrm{p}$.

Identificación de sapogeninas por GC-MS

Se identificaron dos sapogeninas mayoritarias la hecogenina y tigogenina, con porcentajes de área relativa del $51,4 \%$ y $46,1 \%$, respectivamente como se presenta en la Tabla 1.

Tabla 1: Identificación de las señales cromatográficas mayoritarias mediante GC-MS

\begin{tabular}{lllc}
\hline Pico & TR $(\min )$ & Sustancia & $\%$ área relativa \\
\hline 1 & 21,592 & Tigogenina & 46,160 \\
2 & 28,050 & Hecogenina & 51,440 \\
\hline
\end{tabular}

Cuantificación de sapogeninas por HPLC-PDA.

Del barrido para los estándares se encontró que la máxima absorbancia del ácido oleanólico es a $203 \mathrm{~nm}$ y del acetato de hecogenina a $235 \mathrm{~nm}$. Para las muestras se encontraron 2 picos uno a $235 \mathrm{~nm}$ y el segundo a $203 \mathrm{~nm}$.

Los $\mathrm{R}^{2}$ obtenidos para los estándares utilizados fueron de 0,9979 para ácido oleanólico y 0,9984 para acetato de hecogenina, con porcentajes de desviación estándar (\%RSD) de las cinco inyecciones inferiores a 1, tanto para el tiempo de retención como para el área de pico. También los \%RSD fueron menores a 1 para las muestras analizadas, lo que demuestra la confiabilidad estadística de los resultados obtenidos (tabla 2).

El tiempo muerto fue de 4,22 min, de aquí el factor de selectividad resultante fue de 0,94 y la resolución de 1,77 , ésta última se encuentra por encima de 1,5 lo que significa que hay una separación completa de los dos analitos.

El estándar de ácido oleanólico presentó un límite de detección de 1 ppm como traza y el límite de cuantificación fue de $3 \mathrm{ppm}$ a $203 \mathrm{~nm}$. Para el acetato de hecogenina los límites de detección y cuantificación fueron de 4 ppm y 8 ppm respectivamente a $235 \mathrm{~nm}$.

Los análisis por HPLC-PDA permitieron observar los dos picos mayoritarios de sapogeninas con porcentajes en peso similares a las encontradas por GC-MS. En este sentido se cuantificaron 192 
ppm de sapogeninas que correspondieron a 101,5 ppm de hecogenina (52,8 \% en peso) a una longitud de onda de $235 \mathrm{~nm}$ y 90,5 ppm de tigogenina (47,2\% en peso) a $203 \mathrm{~nm}$.

Tabla 2: \%RSD encontrados para área de pico y tiempo de retención para los estándares y las muestras de jugo

\begin{tabular}{|c|c|c|c|c|}
\hline \multirow[t]{2}{*}{ Estándar/muestra } & \multicolumn{2}{|c|}{ \% área relativa } & \multicolumn{2}{|c|}{ Tiempo de retención (min) } \\
\hline & $235 \mathrm{~nm}$ & $203 \mathrm{~nm}$ & $235 \mathrm{~nm}$ & $203 \mathrm{~nm}$ \\
\hline Acetato de hecogenina & 0,040 & - & 0,020 & - \\
\hline Ácido oleanólico & - & 0,031 & - & 0,020 \\
\hline Jugo sin fermentar liofilizado & 0,050 & 0,020 & 0,021 & 0,010 \\
\hline Jugo sin fermentar seco $\left(\mathrm{a} 40^{\circ}\right)$ & 0,028 & 0,050 & 0,031 & 0,042 \\
\hline Jugo a 2 días de fermentación & 0,040 & 0,100 & 0,200 & 0,110 \\
\hline Jugo a 4 días de fermentación & 0,110 & 0,050 & 0,040 & 0,100 \\
\hline Jugo a 6 días de fermentación & 0,040 & 0,100 & 0,050 & 0,280 \\
\hline
\end{tabular}

Los resultados obtenidos para las muestras de jugo de fique fresco y con diferentes tiempos de fermentación se presentan en la tabla 3.

Tabla 3: Contenido de hecogenina y tigogenina en el jugo de fique a diferente tiempo de fermentación

\begin{tabular}{ccccc}
\hline $\begin{array}{c}\text { Tiempo de } \\
\text { fermentación del } \\
\text { jugo (días) }\end{array}$ & $\begin{array}{c}\text { tigogenina } \\
\text { (mg/kg jugo) }\end{array}$ & $\begin{array}{c}\text { hecogenina } \\
\text { (mg/kg jugo) }\end{array}$ & $\begin{array}{c}\text { tigogenina }(\% \mathrm{p} / \mathrm{p}) \\
\text { base seca de jugo }\end{array}$ & $\begin{array}{c}\text { hecogenina }(\% \mathrm{p} / \mathrm{p}) \\
\text { base seca de jugo }\end{array}$ \\
\hline 0 (liofilizado) & 3,297 & 7,196 & 0,008 & 0,018 \\
0 (seco a 40ㄷ) & 2,195 & 4,460 & 0,006 & 0,011 \\
2 & 11,436 & 20,950 & 0,029 & 0,054 \\
4 & 13,938 & 25,390 & 0,035 & 0,063 \\
6 & 6,274 & 10,714 & 0,016 & 0,027 \\
\hline
\end{tabular}

No se encontraron estudios recientes con información sobre la concentración de hecogenina y tigogenina en especies de agaváceas, ya que actualmente las investigaciones están más enfocadas al descubrimiento de nuevas moléculas de saponinas y a su elucidación estructural. Blunden et al. (1978) estudiaron 34 especies y un cultivar de agave, 1 especie de Beschorneria, 1 especie de Dorianthes y 3 especies de Furcraea, entre ellas F. gigantea, encontrando en ésta última, concentraciones de hecogenina y tigogenina de 0,33 y 0,20 (\% peso seco) respectivamente. Los porcentajes en peso seco más altos encontrados en nuestro estudio fueron de 0,063 para hecogenina y 0,035 para tigogenina, con respecto al jugo, el cual representa el $70 \%$ de la hoja, debido al interés de valorizar dicho residuo a través de la extracción de estas sustancias, lo cual podría explicar la diferencia de concentración en sapogeninas en relación a la investigación de Blunden et al. (1978) quienes obtuvieron el extracto a partir de hojas secas y pulverizadas. Es muy factible que el contenido de hecogenina y tigogenina en la hoja de fique sea mucho más alto, ya que se ha observado que los residuos sólidos (bagazo) en contacto con agua generan espuma estable, lo que es indicativo de la presencia de saponinas.

De acuerdo a los resultados anteriores se observa que el jugo de fique presenta mayor contenido de hecogenina en comparación con el de tigogenina, incluso durante el proceso fermentativo. La relación de hecogenina/tigogenina en el jugo fresco es en promedio $6,8 / 3,2$; sin embargo, esta relación disminuye en promedio a $6,4 / 3,6$ durante la fermentación; lo que da a entender que este 
proceso favorece la producción de tigogenina. En la actualidad, la hecogenina presenta mayor uso que la tigogenina, sin embargo un gramo de esta última puede tener un valor hasta 100 veces mayor que el de hecogenina en el mercado farmacéutico, debido a que es un insumo de alta actividad biológica y especificidad (MADR \& MAVDT, 2006). De hecho, un estudio reciente indica que la hecogenina y tigogenina, inducen apoptosis en artritis reumatoide humana, sin embargo, este efecto fue mucho más pronunciado con tigogenina (Liagre et al., 2007). Por otro lado, la tigogenina es precursor en la síntesis de un compuesto que actúa como inhibidor del cáncer de próstata (Merlani et al., 2007).

También se observa que en el jugo de fique se incrementa el contenido de hecogenina y tigogenina desde su estado fresco hasta los 4 días de fermentación, presentando posteriormente un decremento a los 6 días de fermentación.

Biosintéticamente, las saponinas ya sean esteroidales o terpenoides, se obtienen a partir de la vía de los isoprenoides. Todos los terpenoides se derivan de la condensación de 3-isopentenil pirofosfato (IPP) y dimetilalil pirofostafo (DMAPP). Los cuales a su vez se derivan de la condensación de acetil-Co-A. La reacción de condensación de IPP y DMAPP produce geranil pirofosfato (GPP) con 10 átomos de carbono, el cual al unirse con otra unidad de IPP, da origen al farnesil pirofosfato (FPP). Dos unidades de FPP dan lugar al escualeno (C30), cuya subsecuente epoxigenación genera 2,3-oxidoescualeno (C30), el cual es un precursor común de saponinas triterpenoides, fitoesteroles y saponinas esteroidales. Los pasos para la biosíntesis de las saponinas esteroidales no han sido bien elucidados, sin embargo se presume que su precursor inmediato es el colesterol (Augustin et al., 2011).

Se considera que las saponinas y las enzimas involucradas en su biosíntesis hacen parte del sistema defensivo de las plantas (Kohara et al., 2005). Aunque las saponinas son moléculas producidas constitutivamente, su concentración puede aumentar como respuesta a un estímulo externo realizado a sus extractos, tal como quedó demostrado en el trabajo de Lu et al. (2001), en el cual, la concentración de saponinas aumentó cuando el extracto fue sometido a estimulo con levaduras, ácido oligogalacturónico o con metil jasmonato. Lo anterior podría explicar en parte el aumento de concentración de hecogenina y tigogenina del jugo de fique desde el tiempo cero hasta los 4 días de fermentación. Por otro lado la acción de los microorganismos durante el proceso de fermentación del jugo podría también explicar los resultados encontrados, ya que la hidrólisis de las saponinas inicialmente presentes en el jugo produciría un aumento de la cantidad detectada de hecogenina y tigogenina, pero al agotarse el sustrato más fácilmente aprovechable empezaría a presentarse una ruptura o transformación de las sapogeninas, lo que produciría una disminución en su concentración después del cuarto día de fermentación. De hecho, es conocido que el grupo ceto en el anillo $\mathrm{C}$, de la hecogenina, puede ser trasladado a la posición C-11 por métodos químicos o microbiológicos, presentándose una posterior degradación del anillo $\mathrm{F}$, para finalmente, obtenerse hidrocortisona (Osorio, 2009).

\section{CONCLUSIONES}

Se identificaron como hecogenina y tigogenina las sapogeninas mayoritarias presentes en el jugo de fique fresco y fermentado, de la especie Furcraea gigantea proveniente del municipio de El Tambo (Nariño-Colombia).

El mayor contenido de hecogenina y tigogenina se encuentra en el jugo de $F$. gigantea, cuando éste es sometido a fermentación aerobia controlada de cuatro días, en presencia de la flora natural del jugo; por lo cual es recomendable este proceso para la producción de sapogeninas, en especial cuando se busca favorecer la generación de tigogenina.

El jugo de F. gigantea es una interesante materia prima para la obtención de sapogeninas aprovechables en el campo de la farmacología, teniendo en cuenta que éste constituye un residuo que representa el $70 \%$ de la hoja y cuyo volumen de producción en Colombia supera las $450.000 \mathrm{t}$ $a^{-1}$. 


\section{NOMENCLATURA}

$\mathrm{R}_{\mathrm{S}} \quad$ : Resolución de la columna cromatográfica

a $\quad$ : Factor de selectividad de la columna cromatográfica

$\left(t_{R}\right)_{A}$ y $\left(t_{R}\right)_{B} \quad$ : Tiempo de retención de los analitos $A$ y $B$ (sapogeninas)

$W_{A}$ y $W_{B} \quad$ : Ancho de pico cromatográfico para los analitos $A$ y $B$ (sapogeninas)

\section{AGRADECIMIENTOS}

Los autores expresan sus agradecimientos al Ministerio de Agricultura y Desarrollo Rural de Colombia y al Sistema de Investigaciones de la Universidad de Nariño, por el financiamiento de esta investigación.

\section{REFERENCIAS}

Augustin, J., Kuzina, V., Andersen, S., Bak, S., Molecular activities, biosynthesis and evolution of triterpenoids saponins, Phytochemistry: 72, 435-457 (2011).

Barbosa, E. S. Evaluación de la calidad del jugo de fique en la obtención de hecogenina y análisis fitoquímico del extracto heptánico del jugo hidrolizado de Furcraea macrophylla, variedad Negra Común. Tesis de Titulación, Departamento de Química, Universidad Nacional de Colombia Sede Bogotá, Colombia (2002).

Bjarte, Loken, Separation of hecogenin-tigogenin mixtures, US 3935194, clases 435, 465, jan 27 (1976).

Blunden, G., Yi, Y., Jewers, K., Steroidal sapogenins from leaves of Agaveae species, Phytochemistry: 17, 1923-1925 (1978).

Blunden, G., Carabot, A., Jewers, K., Steroidal sapogenins from leaves of some species of agave and furcraea, Phytochemistry: 19, 2489-2490 (1980).

Coleman, J.J., Okoli, I., Tegos, G.P., Holson, E.B., Wagner, F.F., Hamblin, M.R., Mylonakis, E., Characterization of plant-derived saponin natural products against Candida albicans. ACS Chem. Biol.: 5, 321-332 (2010).

De Leo, M., De Tommasi, N., Sanogo, R., D’Angelo, V., Germano, M.P., Bisignano, G., Braca, A., Triterpenoid saponins from Pteleopsis suberosa stem bark, Phytochemistry: 67, 2623-2629 (2006).

Durán, H. y Pulido, J., Análisis de la molienda en el proceso de elaboración de mezcal, Información Tecnológica: 18 (1), 47-52 (2007).

Eskander, J., Lavaud, C., Harakat, D., Steroidal saponins from leaves of Agave macroacantha, Fitoterapia: 81, 371-374 (2010).

Gnoatto, S., Schenkel, E., Bassani, V., HPLC method to assay total saponins in Ilex paraguariensis aqueous extract, J. Braz. Chem. Soc.: 16 (4), 723-726 (2005).

Higgins, J., A high performance liquid chromatographic analysis of the benzoate esters of sapogenins isolated from Agave, J. Chrom.: (121): 329-334 (1976). 
Hostettmann, K. and Marston, A., Saponins: Chemistry and Pharmacology of Natural Products, Cambridge University Press, Cambridge, UK (1995).

Kohara, A., Nakajima, C., Hashimoto, K., Ikenaga, T., Tanaka, H., Shoyama, Y., Yoshida, S., Muranaka, T., A novel glucosyltransferase involved in steroid saponin biosynthesis in Solanum aculeatissimum, Plant Molecular Biology: 57(2), 225-239 (2005).

Liagre, B., Vergne, P., Leger, D., Beneytout, J., Inhibition of human rheumatoid arthritis synovial cell survival by hecogenin and tigogenin is associated with increased apoptosis, p38 mitogenactivated protein kinase activity and upregulation of cyclooxygenase-2, International Journal of Molecular Medicine: 20, 451-460 (2007).

Lu, M., Wong, H., Teng, W., Effects of elicitation on the production of saponin in cell culture of Panax ginseng, Plants Cell Reports: 20(7), 674-677 (2001).

Man, S., Gao, W., Zhang, Y., Huang, L., Liu, C., Chemical study and medical application of saponins as anti-cancer agents, Fitoterapia: 81, 703-714 (2010).

Martinez, A., y Caicedo, T., Bioensayos de toxicidad de jugo de fique en peces en el municipio de El Tambo (Nariño), Tesis de titulación, Dpto. Biología, Universidad El Bosque, Bogotá (2002).

Merlani, M., Amiranashvili, L., Menshova, N., Kemertelidze, E., Synthesis of $5 \alpha$-androstan-3 $\beta, 17 \beta-$ diol from tigogenin, Chemistry of Natural Compounds: 43 (1), 97-103 (2007).

Ministerio de Agricultura y Desarrollo Rural MADR y Ministerio de Ambiente, Vivienda y Desarrollo Territorial MAVDT. Guía Ambiental del Subsector Fiquero. Panamericana Formas e Impresos, Bogotá. Ed. 2, pp. 6-13 (2006).

Montañez, J., Victoria, J., Flores, R., Vivar, M. Fermentación de los fructanos del Agave tequilana Weber Azul por Zymomonas mobilis y Saccharomyces cerevisiae en la producción de bioetanol. Información Tecnológica: 22 (6), 3-14, (2011).

Musende, A.G., Eberding, A., Wood, C., Adomat, H., Fazli, L., Hurtado-Coll, A., Jia, W., Bally, M.B., Guns, E.T., Pre-clinical evaluation of Rh2 in PC-3 human xenograft model for prostate cancer in vivo: formulation, pharmacokinetics, biodistribution and efficacy, Cancer Chemother. Pharmacol.: 64, 1085-1095 (2009).

Oleszek, W. and Bilaly, Z., Chromatographic determination of plant saponins - an update (20022005), J. Chrom.: 1112, 78-91 (2006).

Osorio, E., Aspectos básicos de farmacognosia, Primera edición, pp 1-82, Ed. Universidad de Antioquia, Medellín, Colombia (2009).

Rattanathongkom, A., Lee, J.B., Hayashi, K., Sripanidkulchai, B.-O., Kanchanapoom, T., Hayashi, T., Evaluation of Chikusetsusaponin IVa isolated from Alternanthera philoxeroides for its potency against viral replication. Planta Med.: 75, 829-835 (2009).

Saleem, M., Nazir, M., Ali, M.S., Hussain, H., Lee, Y.S., Riaz, N., Jabbar, A., Antimicrobial natural products: an update on future antibiotic drug candidates. Nat. Prod. Rep.: 27, 238-254 (2010).

San Martin, R., and Briones, R., Industrial uses and sustainable supply of Quillaja saponaria (Rosaceae) saponins, Econ. Bot.: 53, 302-311 (1999).

Sharapin, N., Sapogeninas esteroidales: materia prima para la fabricación de hormonas esteroidales, En Fundamentos de tecnología de productos fitoterapéuticos, Pinzón, R., pp 96-98 Cyted, Colombia (2000). 
Sitton, D., Blunden, G., Cripps, A., Quantitative high-performance liquid chromatographic method for the estimation of hecogenina and tigogenin in the leaves and sapogenin concentrates of Agave sisalana, J. Chrom.: (245): 138-140 (1982).

Skoog, Holler, \& Nieman. Principios de Analisis Intrumental. Quinta Edicion, Mc Graw Hill. (2000).

Sun, S.-X., Li, Y.-M., Fang, W.-R., Cheng, P., Liu, L., Li, F., Effect and mechanism of AR-6 in experimental rheumatoid arthritis, Clin. Exp. Med.: 10, 113-121 (2010).

Tapondjou, L.A., Ponou, K.B., Teponno, R.B., Mbiantcha, M., Djoukeng, J.D., Nguelefack, T.B., Watcho, P., Cadenas, A.G., Park, H.J., In vivo antiinflammatory effect of a new steroidal saponin, mannioside $A$, and its derivatives isolated from Dracaena mannii, Arch. Pharm. Res.: 31, 653-658 (2008).

Yokosuka, A., and Mimaki, Y., Steroidal saponins from the whole plants of Agave utahensis and their cytotoxic activity, Phytochemistry: 70, 807-815 (2009). 